\title{
Epigenetic suppression of the TGF-beta pathway revealed by transcriptome profiling in ovarian cancer
}

\author{
Noriomi Matsumura, ${ }^{1,2,7}$ Zhiqing Huang, ${ }^{1,7}$ Seiichi Mori, ${ }^{3,8}$ Tsukasa Baba, ${ }^{1,2}$ \\ Shingo Fujii, ${ }^{4}$ Ikuo Konishi, ${ }^{2}$ Edwin S. Iversen, ${ }^{5}$ Andrew Berchuck, ${ }^{1}$ \\ and Susan K. Murphy ${ }^{1,6,9}$
}

${ }^{1}$ Department of Obstetrics and Gynecology, Division of Gynecologic Oncology, Duke University Medical Center, Durham, North Carolina 27710, USA; ${ }^{2}$ Department of Gynecology and Obstetrics, Kyoto University, Kyoto 606-8507, Japan; ${ }^{3}$ Institute for Genome Sciences and Policy, Duke University, Durham, North Carolina 27710, USA; ${ }^{4}$ National Hospital Organization Kyoto Medical Center, Kyoto 612-8555, Japan; ${ }^{5}$ Department of Statistical Sciences, Duke University, Durham, North Carolina 27708, USA; ${ }^{6}$ Department of Pathology, Duke University Medical Center, Durham, North Carolina 27710, USA

\begin{abstract}
Epithelial ovarian cancer is the leading cause of death among gynecologic malignancies. Diagnosis usually occurs after metastatic spread, largely reflecting vague symptoms of early disease combined with lack of an effective screening strategy. Epigenetic mechanisms of gene regulation, including DNA methylation, are fundamental to normal cellular function and also play a major role in carcinogenesis. To elucidate the biological and clinical relevance of DNA methylation in ovarian cancer, we conducted expression microarray analysis of 39 cell lines and 17 primary culture specimens grown in the presence or absence of DNA methyltransferase (DNMT) inhibitors. Two parameters, induction of expression and standard deviation among untreated samples, identified 378 candidate methylated genes, many relevant to TGF-beta signaling. We analyzed 43 of these genes and they all exhibited methylation. Treatment with DNMT inhibitors increased TGF-beta pathway activity. Hierarchical clustering of ovarian cancers using the 378 genes reproducibly generated a distinct gene cluster strongly correlated with TGF-beta pathway activity that discriminates patients based on age. These data suggest that accumulation of age-related epigenetic modifications leads to suppression of TGF-beta signaling and contributes to ovarian carcinogenesis.
\end{abstract}

[Supplemental material is available online at http://www.genome.org. The microarray data from this study have been submitted to the NCBI Gene Expression Omnibus (http://www.ncbi.nlm.nih.gov/geo) under SuperSeries accession no. GSE25429.]

\begin{abstract}
Ovarian cancer is the leading cause of death among gynecological cancers. Revealing the factors involved in ovarian carcinogenesis is a top priority in order to develop new modalities for detecting the disease at an early stage (Badgwell and Bast 2007). Epigenetic silencing of gene expression through aberrant methylation of CpG dinucleotides at gene promoter regions plays a major role in carcinogenesis (Barton et al. 2008). Treatment of cells with DNA hypomethylating agents, such as 5-aza-2'-deoxycytidine (decitabine, or 5-Aza-dC) or 5-azacytidine (5-AzaC), followed by gene expression microarray analysis is a frequently used method to detect genes likely to have been silenced by DNA methylation in cancer. A major advantage of this method is that it represents an unbiased genome-wide approach to identify methylation that is relevant to gene expression. A limitation to this approach is that indirect effects cannot be excluded (Esteller 2007). Although extremely high induction of expression was indeed associated with methylated genes in many prior studies, rational strategies have not been developed to discriminate between candidate methylated and unmethylated genes, instead relying on arbitrary cutoffs.
\end{abstract}

\footnotetext{
${ }^{7}$ These authors contributed equally to this work.

${ }^{8}$ Present address: Genomic Oncology Program, Cancer Science Institute Singapore, National University of Singapore, Singapore 117456, Singapore.

${ }^{9}$ Corresponding author.

E-mail murph035@mc.duke.edu; fax (919) 684-5336.

Article published online before print. Article and publication date are at http://www.genome.org/cgi/doi/10.1101/gr.108803.110.
}

Microarray analyses for cells treated with DNA hypomethylating agents have been performed previously using a small number of cell lines, resulting in the identification of relatively few genes that are targeted by DNA methylation. Pathway deregulation resulting from changes in DNA methylation has also not previously been described.

In this study, we treated 39 cell lines and 17 cultured primary ovarian cancer specimens with DNA hypomethylating agents and followed this by microarray analysis to examine genome-wide changes in gene expression. We defined criteria to accurately classify genes as subject to methylation using a list of genes reported as methylated in primary cancers. We used these criteria to identify 378 candidate methylated genes in ovarian cancer and showed for the first time that TGF-beta pathway function is regulated by methylation of multiple TGF-beta pathway gene members in this disease. Gene expression analyses of the 378 candidate methylated genes in primary ovarian cancer tissue specimens suggested that a defined cluster of genes is associated with suppression of TGFbeta pathway activity though age-related coordinate accumulation of methylation. These data suggest a fundamental mechanism underlying the development of ovarian cancer.

\section{Results and Discussion}

Prediction of methylated genes in ovarian cancers

We generated gene expression microarray data for 39 ovarian cancer cell lines that were either mock treated or treated with 
5-Aza-dC. Unsupervised hierarchical clustering of the resulting data indicated that the 5-Aza-dC treatment did not cause nonspecific genome-wide changes in gene expression (Supplemental Fig. S1).

Most of the currently known methylated genes in ovarian cancer (Barton et al. 2008) are also methylated in other types of cancers. We found a very similar pattern of gene reactivation in multiple cell lines from different tissue sources by pharmacologic reactivation of expression using DNA methyltransferase inhibitors (Supplemental Table S1; Supplemental Fig. S2), also suggesting commonality in genes targeted by DNA methylation across tissues. We previously used a compilation of genes reported as methylated in cancers in a bioinformatics approach to predict genes subject to methylation in ovarian cancer based on sequence context (Goh et al. 2007). In the current study, we used the same list (Supplemental Table S2) for validating our methodology in the prediction of methylated genes. Because genes methylated in other types of cancers are more likely to be methylated in ovarian cancer than are other genes throughout the genome, we searched for parameters that optimally enrich for this group of genes, which we refer to as "methylated in cancer" genes (MIC), which could then be used to predict genes methylated in ovarian cancer.

Previously, high induction of expression following 5-Aza-dC treatment in a panel of cell lines has been used to predict methylated genes (Muthusamy et al. 2006; Hoque et al. 2008). When all microarray probes were sorted by their maximal change (MaxC) in gene expression among the 39 ovarian cancer cell lines, the MIC genes were enriched, strongly suggesting that highly reactivated genes are methylated in ovarian cancer. In our analysis, the frequency in occurrence of genes from the MIC list showed an abrupt upward shift at a MaxC of 2.9 (Fig. 1A). This enabled us to empirically determine a threshold for MaxC, above which a gene has an increased likelihood of exhibiting methylation. Because MaxC was calculated by subtraction of $\log _{2}$ RMA (robust

A

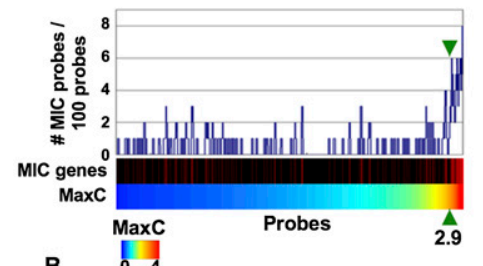

B $\quad 04$

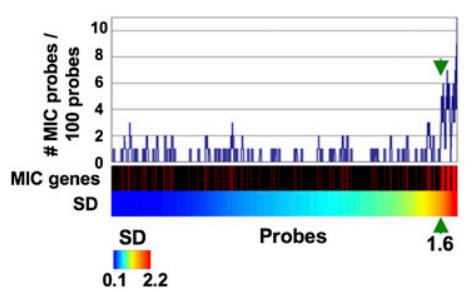

Figure 1. Defining threshold criteria for prediction of methylation. Analysis of expression of the MIC genes from ovarian cancer cell lines for maximum change (MaxC) in expression following 5-Aza-dC treatment relative to mock-treated cells (A), for the standard deviation (SD) in expression among the mock-treated cells $(B)$, and for the combination of these two parameters $(C)$. In panels $A$ and $B$, gene probes are arranged in increasing order of $\operatorname{Max} C(A)$ or $\operatorname{SD}(B)$, indicated by the color bar at the bottom of each panel. The number of MIC genes represented in a 100-probe sliding window is indicated by the blue bar graphs. The positioning of MIC genes with respect to MaxC or SD is shown in the color bar below, with red lines representing MIC genes and other genes represented by black lines. Defined thresholds are indicated by the green arrows. In the scatter plot in panel $C$, each gene probe is represented by a single dot where $x=\mathrm{SD}$ and $y=$ MaxC. (Red dots) MIC genes; (black dots) all other genes.

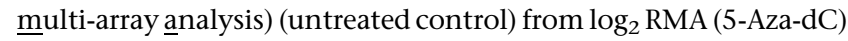
values, this threshold of 2.9 is comparable to previous reports that detected methylation of genes exhibiting a 4 - to 8 -fold induction (Muthusamy et al. 2006; Shames et al. 2006). Although this criterion was extremely stringent when cell lines were considered individually, 763 probes (623 genes) were selected based on analysis entire group of 39 cell lines. Therefore, we looked for another arameter to increase selectivity for MIC gene enrichment.

An unexpected factor that enriched MIC genes was the standard deviation (SD) of gene expression among the mock treated 39 cell lines, which is completely independent from the MaxC parameter that relies on induction of expression following drug treatment. The enrichment of MIC genes abruptly shifted upward at the point where the SD was greater than1.6 (Fig. 1B), and this sudden increase again provided an empirically defined SD threshold above which genes are more likely to exhibit methylation. Similar results were obtained for two other primary ovarian cancer microarray data sets (Supplemental Fig. S3). Although not previously reported, this data indicates that aside from changes in copy number, large differences in gene expression that likely contribute to the heterogeneous phenotypes of cancer cells may also be due to differences n epigenetic gene regulation. One limitation of using a high SD to identify candidate methylated genes is that this may inhibit identification of those genes that are very frequently silenced by methylation, since expression levels for such genes are expected to be ubiquitously low. However, combining the MaxC and SD parameters led to synergistic enrichment of MIC genes and produced list of 311 candidate methylated genes (379 probes) (Fig. 1C;

Methylation microarrays have been used to directly detect genome-wide methylation based on digestion of genomic DNA with methylation-sensitive and insensitive restriction enzymes, or through capture of methylated DNA fragments using methylDNA binding proteins, ultimately leading to generation of an unbiased list of methylated genes. We therefore also compiled a methylation microarray gene list from such published reports (Supplemental Table S3). We found that the cutoff values for both MaxC $>2.9$ and SD > 1.6 significantly enriched the genes from the methylation microarray gene list in the cell lines (Supplemental Table S4; $P<0.0001$ for both, $\chi^{2}$ test) as we had also observed for the genes in the MIC gene list. Exclusion of the MIC genes did not change the significance of the results for the remaining methylation microarray gene probes (data not shown). Furthermore, we analyzed the ability of the MaxC and SD parameters to enrich for the genes predicted to be methylated based on sequence context from our prior report (Goh et al. 2007). In this report, genes likely to be methylated were assigned higher cluster_boost scores. Indeed, genes with higher cluster boost scores were enriched above the cutoff values of MaxC > 2.9 and SD > 1.6, independently (Mann-Whitney $U$ test, $P=0.012$ and $P<0.0001$, respectively; not shown). These results, from analysis of mulitiple genome-wide, unbiased gene 
Table 1. High MaxC and high SD enrich MIC genes

\begin{tabular}{llll}
\hline Cell lines & \multicolumn{1}{c}{ SD $>1.6$} & \multicolumn{1}{c}{ SD $<1.6$} & \multicolumn{1}{c}{ Total } \\
\hline MaxC $>2.9$ & $7.7 \%(29 / 379)$ & $1.8 \%(7 / 384)$ & $4.7 \%(36 / 763)$ \\
MaxC $<2.9$ & $3.1 \%(21 / 674)$ & $0.4 \%(84 / 20,778)$ & $0.5 \%(105 / 21,452)$ \\
Total & $4.7 \%(50 / 1053)$ & $0.4 \%(91 / 21,162)$ & $0.6 \%(141 / 22,215)$ \\
\hline
\end{tabular}

Shown is the percent of probes meeting each criteria, followed by the actual ratio in parentheses, which shows the number of probes in the MIC data set meeting the specified criteria divided by the number of probes on the gene chip that also meet the criteria. For example, $7.7 \%$ of the probes representing the MIC genes exhibit an average SD $>1.6$ and an average MaxC of $>2.9 ; 7.7 \%$ is derived by dividing the $29 \mathrm{MIC}$ gene probes by the 379 probes on the Affymetrix U133A platform that also meet these same criteria for SD and MaxC and multiplying that number by 100 .

sets and based on known methylation-prone genes in cancers, validate the prediction of methylated genes using our parameters of MaxC and SD.

Candidate genes identified from reactivation-microarray experiments using cell lines are thought to require independent validation using clinical samples (Cairns 2009). However, others have shown that most genes methylated in cell lines are also subject to methylation in clinical cancer samples (Ueki et al. 2002; Ibanez de Caceres et al. 2006; Muthusamy et al. 2006; Shames et al. 2006). To enable comparison of established cell lines with clinical cancer samples, we developed a primary culture data set, consisting of 13 ovarian cancers (12 serous, one endometrioid), two serous borderline tumors, and two pooled normal ovarian surface epithelium samples (Supplemental Table S5). These specimens underwent limited culture (one passage) followed by mock treatment or treatment with $5 \mu \mathrm{M}$ 5-AzaC. RNA from these cells was subsequently used for generation of gene expression microarray data. As we had observed with the cell lines, both MaxC induced by 5-AzaC and the SD of expression among the untreated samples enriched for the MIC genes. The number of MIC genes sharply increased with parameters of MaxC > 1.5 and SD > 1.6, which represented distinct thresholds for this group of specimens (Supplemental Fig. S4A,B). Similar to the cell lines, synergistic enrichment of MIC genes occurred when the two criteria were combined, and identified 108 candidate methylated genes (128 probes) (Supplemental Fig. S4C; Supplemental Table S6). These cutoff values also enriched the methylation microarray gene list (Supplemental Table S7; $P<$ 0.0001 for both). Furthermore, higher cluster_boost scores (Goh et al. 2007) were again observed among the genes selected by the two cutoff values $(\mathrm{MaxC}>1.5 ; P=0.0061, \mathrm{SD}>1.6 ; P<0.0001$; data not shown).

Next, we compared the selected candidate methylated genes in the cell line and primary culture data sets. Many of the probes selected from the primary cultured cells were present among the genes exceeding the MaxC and SD cutoffs defined by the cell line gene expression profiles (Fig. 2A). Similarly, the MaxC and SD thresholds defined by the primary cultured cells clearly enriched the probes selected from the cell lines (Fig. 2B). These data indicate that candidate methylated genes exhibit similarities in expression patterns among established cell lines and clinical samples in ovarian cancer. Compared to the 311 candidate methylated genes (379 probes) from the cell line data, only 108 candidate methylated genes (128 probes) were identified from the primary cultures, probably due to the smaller sample size as we alluded to above from individual analysis of the cell lines. There were 41 genes that were present in both gene lists, which was statistically significant compared to the rest of the genome $\left(P<0.0001, \chi^{2}\right.$ test). Given the degree of overlap between the cell line and primary culture data sets, both gene lists were combined and comprised our final group of 378 candidate methylated genes (454 probes) in ovarian cancer (Supplemental Table S8).

The reliability of these predictions was also assessed using a literature search. Among the 378 predicted methylated genes, 167 (44\%) were previously reported as methylated inclusive of all cell types, among which 21 were in ovarian cancer and 127 in other types of malignant cells (Supplemental Table S8). Only 14 of the 167 genes that were previously reported as methylated in any cancer were also members of the MIC gene list. Our ratio of known methylated/predicted methylated $(167 / 378)$ is much higher than that from a previous report $(25 / 200 ; 12.5 \%)$ in which 20 cell lines were used in a gene reactivation-microarray experiment (Hoque et al. 2008) $\left(P<0.0001, \chi^{2}\right.$ test $)$, though this difference may be at least partially influenced by the different timing of the literature analysis. Based on these observations, we anticipated that many of the 378 listed candidates from our analyses are bona fide methylated genes.

In order to biologically verify the predictions, we conducted methylation-specific PCR assays and pyrosequencing analysis. Methylation-mediated regulation of transcription does not necessarily occur at $\mathrm{CpG}$ islands that contain transcription start sites (TSS-CGI) (Antequera 2003). However, here we analyzed only
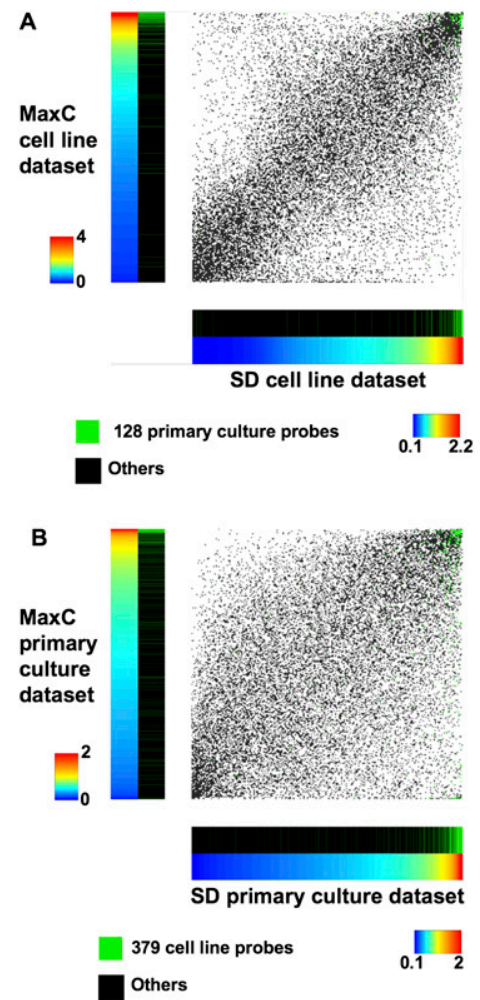

Figure 2. Concordance between predictions from primary cultured cell and cell line data of candidate methylated genes. (A) Enrichment of the 128 candidate methylated gene probes (green lines in black color bar; other probes, black lines; in scatter plot, green dots) from primary cultured cancer cells based on the thresholds for MaxC and SD defined by analysis of the cell line data. (B) Enrichment of the 379 candidate methylated gene probes from the cell line data set (in black color bar, green lines; other probes, black lines; in scatter plot, green dots; other probes, black dots) based on thresholds for MaxC and SD defined by the primary culture data set.

\section{Genome Research} www.genome.org 
TSS-CGI(+) genes because we presumed this would provide the most straightforward means to detect DNA methylation that regulates transcription and thus reflects our defined MaxC and SD parameters. For reasons described later, TGF-beta pathway-related genes were a dominant focus of these analyses. We analyzed 43 genes, including two that have been reported as methylated in ovarian cancer (see Supplemental Table S8). All 43 genes were found to be methylated in at least three of the ovarian cell lines tested. Furthermore, we found methylation of ten additional genes, selected by either high MaxC or high SD, in the ovarian cell lines and among the ovarian cancer primary culture specimens. Among these 53 genes, 50 were also analyzed in primary ovarian cancer tissues and 48 genes were methylated in at least one of the cancer specimens analyzed (see Supplemental Table S9 and Supplemental Fig. S5A for representative MS-PCR results). Furthermore, these 50 genes were also examined in primary ovarian cancer cell cultures and 46 genes exhibited methylation in at least one specimen (Supplemental Table S9). These data strongly suggest that most, if not all, of the predicted genes are actually targeted by DNA methylation in ovarian cancer.

Next, we analyzed the relationship between methylation and expression for the methylation prone genes in the ovarian cell lines. Relative transcript levels of genes that exhibit methylation tended to be lower than that of specimens that were unmethylated when analyzed by MS-PCR. This association was statistically significant for 22 genes ( $P<0.05$; Supplemental Fig. S5B). To further verify the inverse relationship between expression and methylation, TGFBR2, THBS1, and ID1 were analyzed using pyrosequencing, which provided highly quantitative data for individual CpG sites in the promoter regions of these genes. Significant negative correlations between expression and methylation were found for these genes (Fig. 3; Supplemental Fig. S5C). These data indicate that prediction of methylated genes based on our method, using expression microarray data from established cell lines and primary cultured cells, identifies genes for which expression is subject to suppression by DNA methylation.

A

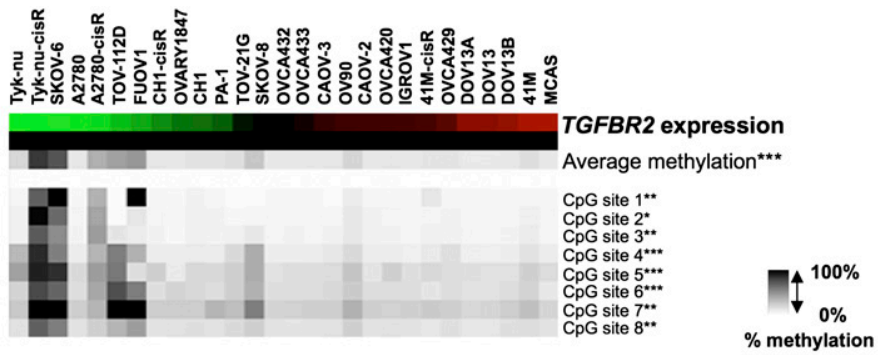

B

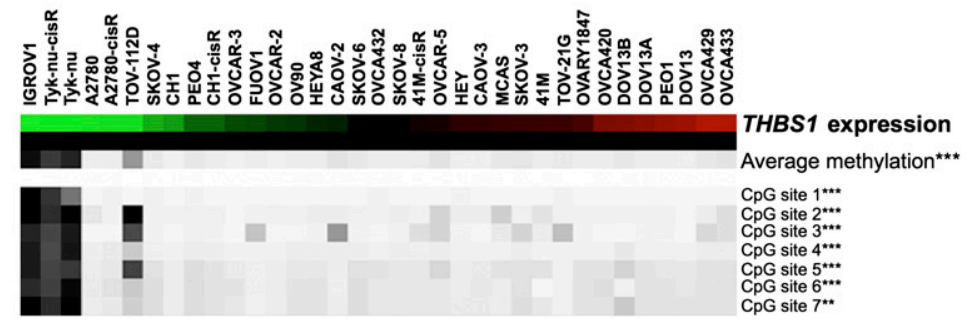

Figure 3. Methylation of predicted genes is associated with repression of transcription. Quantitative pyrosequencing was used to measure methylation present at the promoters of TGFBR2 (8 CpG sites; $A$ ) and THBS1 (7 CpG sites; $B$ ). Expression is indicated by the green-red (low-high) color bars at the top of each panel. Significant correlations between methylation and expression are indicated by the asterisks: ${ }^{*} P<0.05 ;{ }^{* *} P<0.01 ;{ }^{* *} P<0.001$.

\section{Pathway analysis of the predicted methylated genes}

Treatment of cells with inhibitors of DNA methyltransferase activity is expected to result in increased expression of genes that are silenced by DNA methylation, which can be comprehensively detected using microarrays (Ushijima 2005). By extension, it is plausible that signaling pathways regulated by DNA methylation are more likely to be revealed using this technique than with other methods that directly detect genome-wide methylation. The interferon signaling pathway is up-regulated by 5 -Aza-dC in colon cancer (Karpf et al. 1999) and bladder cancer (Liang et al. 2002). However, very few reports have analyzed associations between methylation and signaling pathways likely because the number of methylated genes detected by such reactivation-microarray experiments has been relatively small (Mund et al. 2006). Here, we generated a list of 378 genes predicted as subject to methylation in ovarian cancer by using as many as 56 specimens with the anticipation that the larger sample size would allow us to more effectively detect pathway-specific regulation by DNA methylation.

To better understand the biological relevance of methylation in ovarian cancer, the potential enrichment of Gene Ontology (GO) terms for the 378 predicted genes was assessed using GATHER (Supplemental Table S10; Chang and Nevins 2006). Consistent with the widely accepted idea that gene-specific methylation in cancer targets genes with tumor suppressive functions and contributes to cancer initiation, GO terms such as "negative regulation of cell proliferation" $(P<0.001)$ and "positive regulation of apoptosis" $(P<0.01)$ were significantly enriched among the 378 predicted methylated genes as compared to the genome. GO terms including "development" and "morphogenesis" were also enriched and are consistent with previous reports that DNA methylation is essential in the physiological process of development (Suzuki and Bird 2008). Other representative GO terms that were enriched in this gene set included "adhesion," "cell migration," "angiogenesis," and "immune response." These GO terms, which are relevant to the functions of the TGF-beta superfamily pathway (Massague 2008), are suggestive of a close relationship between DNA methylation and this pathway. In support of this, we used GATHER to analyze the gene list for potential enrichment of genes belonging to KEGG pathways (Kyoto Encyclopedia of Genes and Genomes, http://www.genome.ad.jp/kegg/), and found that genes belonging to the "TGF-beta signaling pathway" were indeed significantly enriched among the 378 genes compared to the genome $(P=$ $0.003)$.

Deregulation of the TGF-beta signaling pathway has been detected in ovarian cancer. Mutation of TGFBR2 has been reported (Lynch et al. 1998), but the frequency is thought to be very rare (Alvi et al. 2001; Francis-Thickpenny et al. 2001; Ding et al. 2005). In accordance with this, several groups including ours have reported that TGF-beta 1-induced post receptor signal transduction occurs in most ovarian cancer cells. However, the tumor suppressive function of 
TGF-beta is decreased or diminished, and its prometastatic function is increased, although the precise mechanism remains unclear (Havrilesky et al. 1995; Rodriguez et al. 2001; Dunfield et al. 2002; Do et al. 2008). In human cancers, promoter methylation of genes within the TGF-beta signaling pathway has been reported (Kang et al. 1999; Li et al. 1999; Zhao et al. 2005; Furuta et al. 2006; Onwuegbusi et al. 2006). Other TGF-beta superfamily signaling genes are also targeted by methylation (Umetani et al. 2005; Wen et al. 2006; Yamashita et al. 2006). Although methylation of TGFbeta superfamily signaling pathway genes has never been reported in ovarian cancer, it is plausible that this is one of the major pathways that would be targeted by promoter methylation given the enrichment of the GO terms relevant to this pathway. To verify this idea, we first analyzed methylation of 12 KEGG TGF-beta superfamily genes in ovarian cancer samples using MS-PCR. BMP2, BMP4, FST, ID2, ID4, TGFB2, and THBS1 were included among the 378 predicted methylated genes. $B M P 7, I D 1, I N H B B, S M A D 7$, and TGFBR2 were also selected as potentially methylated using either the high MaxC or high SD criteria when applied to the cell line or primary culture data sets (Supplemental Table S9). All 12 selected genes exhibited methylation in both ovarian cell lines and cancer tissue samples.

Next, we examined the associations between the results of genome-wide demethylation treatment using 5-Aza-dC and TGFbeta activity. In the SKOV4 and OVCAR3 ovarian cancer cell lines, 5-Aza-dC enhanced TGF-beta activity, detected by Western blotting using antibodies against p-SMAD3. Interestingly, the activation resulting from 5-Aza-dC treatment appeared stronger than that from the treatment by TGF-beta 1 alone in the SKOV4 cells (Fig. 4A). This trend was more evident using a luciferase assay with a SMAD3-reporter plasmid (Fig. 4B).

To further confirm the induction of TGF-beta pathway activity by 5 -Aza-dC, we conducted binary regression, a computational method used to calculate pathway activity based on the change in transcriptional activity of downstream pathway genes (West et al. 2001; Bild et al. 2006). We previously developed and reported a TGF-beta pathway signature using a data set available from the Gene Expression Omnibus website (GSE1724; http:// www.ncbi.nlm.nih.gov/geo/; Renzoni et al. 2004; Andrechek et al. 2008). Consistent with the results obtained from Western blotting and the luciferase assay, this computational analysis indicated that 5-Aza-dC treatment significantly induced TGF-beta pathway activity (Supplemental Fig. S6; Figure 4C).

In order to quantitatively assess the genome-wide intensity of epigenetic gene silencing and relate this to TGF-beta pathway activity, we first calculated the average expression values of the 378 genes (454 probes), a method used previously to calculate gene signature expression profiles of tumors determined by a specific gene set (Chen et al. 2007). In the 39 untreated cell lines, TGF-beta signature probabilities were strongly positively correlated with the average expression of the 378 genes $(r=0.89, P<0.0001$; signature probabilities are listed in Supplemental Table S11). Because TGF-beta pathway function was induced by 5-Aza-dC treatment, these results suggest that one of the major effects of the cumulative genome-wide DNA methylation profiles in ovarian cancers is to suppress TGF-beta pathway activity. In addition, correlation analyses with cell line characteristics with relevance to TGF-beta pathway activity, including anchorage-independent growth, population doubling time, and cisplatin response, suggest methylation-mediated inhibition of tumor suppressor functions (Supplemental Table S11; Supplemental Fig. S7). The average expression of the 378 genes negatively correlated with the methyla-
A

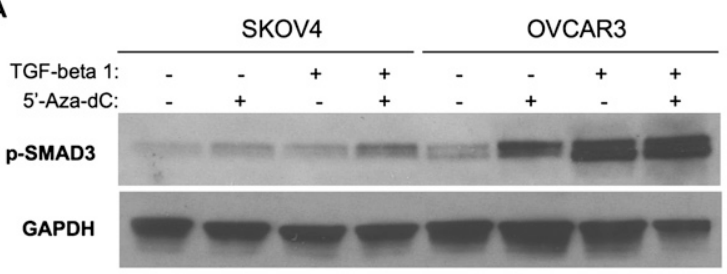

B
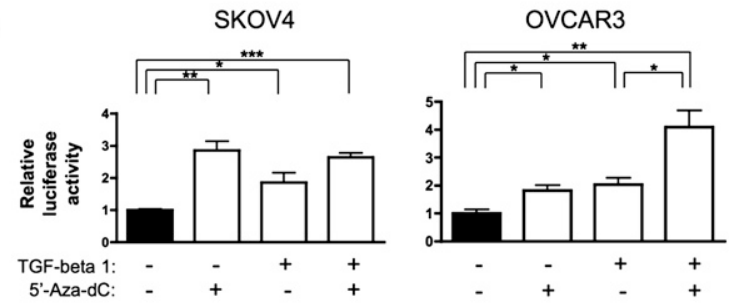

C

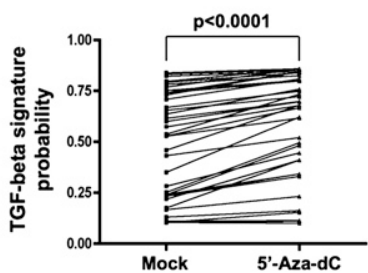

Figure 4. The TGF-beta pathway is activated following treatment with DNA hypomethylating agents. (A) Representative Western blot showing that levels of the TGF-beta effector molecule, phosphorylated SMAD3 (p-SMAD3), increase following addition of 5-Aza-dC or TGF-beta 1 in the SKOV4 and OVCAR3 ovarian cancer cell lines. GAPDH was used as an endogenous control. Combined treatment leads to synergistic induction of p-SMAD3. Similar results were obtained using a SMAD3 reporter luciferase assay $(B)$. The average of three experiments is shown. Error bars, SD. ${ }^{*} P<0.05,{ }^{*} P<0.01,{ }^{* *} P<0.001$. (C) TGF-beta pathway signature probability scores are increased in 39 cell lines following treatment with 5-Aza-dC.

tion status of the entire group of 53 genes in Supplemental Table S9 (Supplemental Fig. S8; $r=-0.61, P=0.0002$ ), supporting the relevance of using the average expression of the 378 genes to represent genome-wide methylation status.

We next analyzed the clinical ovarian cancer data set, GSE3149, which contains 146 tissue samples from advanced (stage III-IV) serous ovarian cancers without prior treatment. Hierarchical clustering analysis using our group of 378 genes generated one pronounced gene cluster in which the expression of the genes was strongly correlated (Fig. 5A). To analyze the reproducibility of the cluster classification, we used GSE2109 containing gene expression microarray data for over 2000 tumor specimens, from which the 77 serous ovarian tumor samples without any prior treatment were analyzed. These 77 specimens included borderline tumors and early and advanced stage cancers. The same probes that exhibited highly correlated expression in GSE3149 again generated a gene cluster in the GSE2109 data set (Fig. 5B). Although we cannot formally rule out similarity in copy number changes, alterations in a common upstream regulatory factor or histone modifications in contributing to this clustering pattern, these data likely suggest that concordant epigenetic regulation of transcription occurs for a substantial number of genes in serous ovarian cancers and that this is present in premalignant tumors and early stage disease. We refer to the genes that are present in the coordinately expressed gene cluster as "methyl cluster" genes. 
A

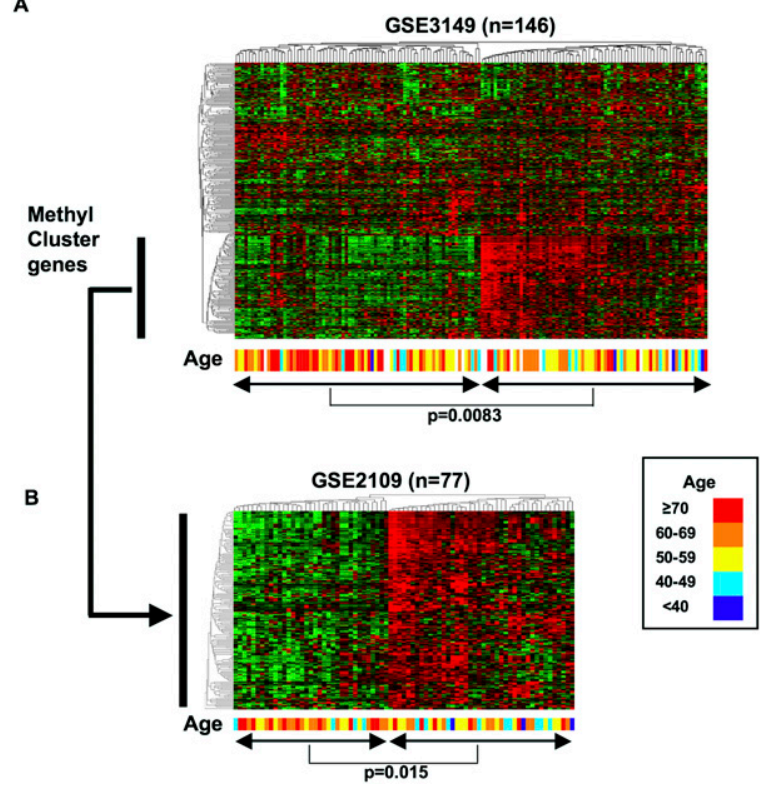

Figure 5. Methyl cluster gene suppression is associated with age. $(A)$ A defined gene cluster (designated as "methyl cluster") was revealed through hierarchical clustering of 146 primary ovarian cancers based on the expression of the 378 candidate methylated genes (454 probes). Expression is shown by green to red (low to high) coloration within the heatmap with age of the patient (below the heatmap) inversely correlated with expression. (B) The methyl cluster genes in panel $A$ similarly stratified an independent set of 77 primary ovarian cancers, with lower gene expression again associated with increased age.

In the data set containing 146 advanced stage serous ovarian cancers (GSE3149), survival of patients did not differ between the two tumor clusters stratified by the methyl cluster genes (data not shown). However, in the tumor cluster in which the expression of the methyl cluster genes was suppressed, the patient's age at diagnosis was significantly higher (mean; $62.6 \mathrm{yr}$ vs. $57.7 \mathrm{yr}, P=$ 0.0083; Mann-Whitney $U$ test) (Fig. 5A). In the data set containing 77 serous ovarian tumors (GSE2109), there was no difference in the overall distribution of the borderline tumors, early stage cancers, and late stage cancers among the two tumor clusters based on expression of the methyl cluster genes (data not shown). However, the patient's age at diagnosis was again significantly higher in the group with repressed expression of the methyl cluster genes (mean; 63.6 yr vs. $56.7 \mathrm{yr}, P=0.015$; Fig. $5 \mathrm{~B}$ ).

The average expression of the 378 genes was positively correlated with the expression of genes in the methyl cluster $(P<$ 0.0001; data not shown) indicating that expression of the methyl cluster genes is representative of the methylation target genes. A strong positive correlation was also observed between the TGFbeta signature probabilities and the average expression of the methyl cluster genes in both GSE3149 and GSE2109 (Fig. 6A,B; $P<0.0001$ for both; TGF-beta signature probabilities are listed in Supplemental Table S12). This relationship was also present in the data sets for the 39 ovarian cell lines and the 17 primary cultured cells (Fig. 6C,D; $P<0.0001$ for both). The two pooled OSE specimens showed high expression of the methyl cluster genes and high TGF-beta signature probability in the primary culture data set (Fig. 6D). Distribution of the two borderline specimens did not show any specific pattern of distribution in the primary culture data set (data not shown).
Several genes that encode gene products involved in activation of TGF-beta pathway signaling are present within the methyl cluster (Supplemental Table S8), including THBS1, CD44, and SDC2. Though TGFBR2 was not included in the 378 predicted genes, it was methylated in ovarian cancer cells (Fig. 3A) and showed a strong positive correlation with the TGF-beta signature probability in the ovarian cancer data set GSE3149 $(r=0.49, P<$ $0.0001)$. Therefore, these genes may exhibit concordant epigenetic deregulation in ovarian cancer, and this may contribute to the suppression of TGF-beta activity. Furthermore, genes downstream from the TGF-beta signaling pathway are also present in the methyl cluster genes. SNAI2, TGFBI, SERPINE1, TAGLN, CTGF, and EGR1 are well-known targets of TGF-beta signaling and mediate epithelial-mesenchymal transition (EMT) or produce extracellular matrix (Ihn 2002; Hirashima et al. 2003; Chen et al. 2006; Herfs et al. 2008; Ma et al. 2008; Medici et al. 2008; $\mathrm{Yu}$ et al. 2008). In our study, methylation of all of these genes was detected in ovarian cancers (Supplemental Table S9; Supplemental Fig. S5). Furthermore, epigenetic regulation of other genes within the group of 378 candidate methylated genes might also influence TGF-beta pathway activity through unknown mechanisms.

Tothill et al. (2008) defined six transcriptional subtypes (C1C6) from microarray analysis of serous and endometrioid ovarian cancers (Tothill et al. 2008). Of these, C1 tumors (high stroma signature) displayed high levels of tissue desmoplasia, which is relevant to TGF-beta pathway activity. The average expression of both the 378 candidate methylated genes and the methyl cluster genes was highest in the C1 tumor group (Supplemental Fig. S9; ANOVA $P<0.0001$ ). The C4 tumor group (low stroma signature) showed significantly lower average expression of these genes as compared to the $\mathrm{C} 1$ tumor group ( $P<0.0001$; unpaired $t$-tests). Therefore, the recently defined transcriptional subtypes seem to be at least partially attributed to genome-wide methylation status.

Taken together, our results indicate that concordant epigenetic silencing is more likely to occur with age and may contribute to the molecular heterogeneity present in ovarian carcinogenesis. The relatively high expression of the Methyl Cluster genes and the high TGF-beta signature probabilities of the pooled primary cultured normal OSE (Fig. 6D) suggests that the genome-wide accumulation of DNA methylation modulates ovarian cancer phenotypes at least in part through suppression of TGF-beta activity. Age-related accumulation of gene-specific methylation has been reported in relation to increased risk or presence of prostate and colon cancers (Ahuja et al. 1998; Kwabi-Addo et al. 2007) and our data suggest that this may also apply to ovarian carcinogenesis. However, ovarian tumors with age-related increases in methylation appear to not be associated with a worse prognosis, nor are they more invasive. This is likely due to the biphasic functions of TGF-beta in suppressing cell proliferation and also in promoting the aggressiveness of ovarian cancer (Nilsson and Skinner 2002).

In summary, transcriptome profiling using gene expression microarrays following pharmacologic reactivation of genes targeted by DNA methylation in 39 ovarian cell lines and 17 primary culture samples led to defining two threshold values for MaxC and SD which generated a list of 378 candidate methylated genes in ovarian cancer. From analysis of those genes, a close relationship between the genome-wide DNA methylation profile in ovarian cancers and TGF-beta pathway activity was revealed. Furthermore, we suggest that this concordant epigenetic deregulation leads to classification of ovarian cancers into the following two subgroups: 


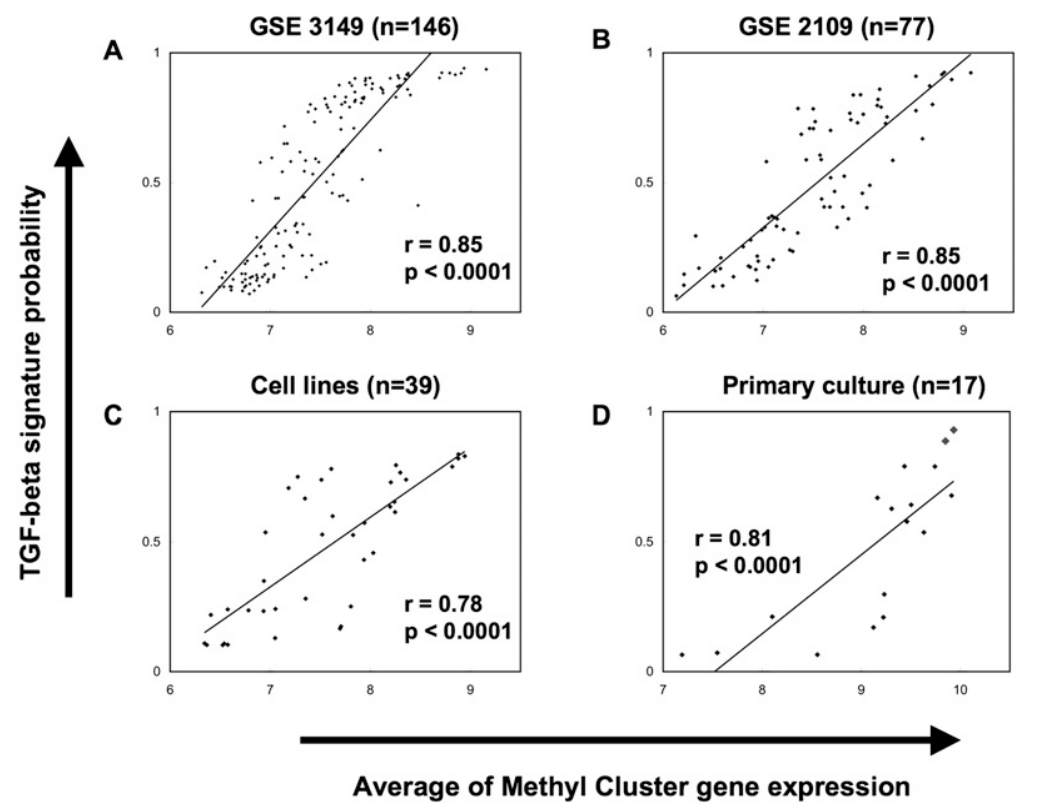

Figure 6. Correlation between TGF-beta signature probability and methyl cluster genes. The TGFbeta signature probability in ovarian cancer is strongly positively correlated with the average expression of the methyl cluster genes in 146 primary ovarian cancers $(A)$, an independent set of 77 primary ovarian cancers $(B)$, in the 39 established cell lines studied $(C)$, and in the 17 primary cultured ovarian specimens $(D)$. Diamond symbols in panel $D$ represent the two pooled normal ovarian surface epithelium specimens.

(1) hypermethylated tumors with suppressed TGF-beta pathway activity in women of increased age, and (2) tumors with increased TGF-beta pathway activity in younger women. Epigenetic therapy (Yoo and Jones 2006) may be useful for the former group, and conversely, TGF-beta inhibitor therapy (Massague 2008) may be useful for the latter group. Our data highlight the utility of transcriptome reactivation microarray analysis to reveal how altered epigenetic regulation modulates signal pathways in cancer, and therefore, links the biology of epigenetics to future translational research in cancer.

\section{Methods}

Cell culture, in vitro experiments, and methylation assays.

Detailed methods are described in the Supplemental text. We previously reported the list of the 39 cell lines used in this study and their culture conditions (Baba et al. 2009; Kondoh et al. 2010). Population doubling times, colony formation in methylcellulose, and cisplatin IC50 values were determined for all cell lines. Cell lines were treated with $5 \mu \mathrm{M} 5$-Aza-dC or mock treated for $72 \mathrm{~h}$ prior to microarray analysis. Western blotting and luciferase assays were performed using SKOV4 and OVCAR3 ovarian cancer cell lines to examine TGF-beta pathway activity in vitro. All tissue specimens were collected after each patient provided written informed consent and were used under a protocol reviewed and approved by the Duke University Institutional Review Board. Primary culture of 13 ovarian carcinoma, two borderline tumors, and ovarian surface epithelium (Supplemental Table S5) was performed as previously described (Lounis et al. 1994). Cells in primary culture were divided into two dishes, mock treated or treated with $5 \mu \mathrm{M} \mathrm{5-AzaC}$ for $72 \mathrm{~h}$. Methylation analyses were performed using methylation-specific (MS)-PCR and pyrosequencing using methods previously reported by our group (Huang et al. 2006) and described in the Supplemental text. Primers for MS-PCR analysis are listed in Supplemental Table S13.

\section{Microarray data sets}

Gene expression microarray data was generated as previously reported (Berchuck et al. 2005). RNA was processed and hybridized onto Affymetrix HT-U133A (cell lines) or U133 Plus 2.0 (primary culture) genes chips by the Duke Microarray Facility. Published microarray data sets were obtained from the Gene Expression Omnibus website (http://www. ncbi.nlm.nih.gov/geo/) and included GSE4717, GSE5230, GSE5816, GSE1724, GSE6653, GSE5457, GSE7144, GSE3149, GSE9899, and GSE2109. Microarray data sets previously described but not made publically available were generously provided by $\mathrm{T}$ Ushijima (Yamashita et al. 2006), Dr. Suzuki (Suzuki et al. 2002), and JC Keen (Keen et al. 2004). A list of genes known to be methylated in cancers (MIC genes) was assembled from the MD Anderson website (http://www.mdanderson. org/departments/methylation/) in addition to literature searches as we have previously described (Goh et al. 2007). Methylated genes as detected using methylation microarrays were obtained from published papers (see Supplemental Table S3).

\section{Data mining of microarray data sets}

Graphs for the frequency of MIC genes in the neighboring 100 probes (Fig. 1A,B; Supplemental Fig. S4A,B) were drawn using Excel. Software applications were downloaded from the following websites: R, http://www.r-project.org; Java TreeView version 1.1.2, http://jtreeview.sourceforge.net/; Cluster 3.0, http://rana.lbl.gov/ EisenSoftware.htm; Binary regression, http://data.genome.duke. edu/oncogene.php. The enrichment of Gene Ontology terms or KEGG pathways within groups of genes or gene probes was performed using web-based GATHER (http://gather.genome.duke.edu/) (Chang and Nevins 2006). Blue-yellow-red heatmaps were drawn using R, and other heatmaps were drawn using Java TreeView. Average linkage hierarchical clustering was conducted using mean centered and normalized gene expression values. Statistical analysis was performed using GraphPad Prism Version 4.0b. Binary regression analysis used MAS5 normalized values. All other microarray data set analyses used $\log _{2}$-transformed RMA-normalized values. Of the more than $54,000 \mathrm{U} 133$ Plus 2.0 probes, only the 22,215 that were also present in the U133A data set were used. To merge U95A V2 gene chips with U133A probes, the "bestmatch" annotations were obtained from the Affymetrix website (http:// www.affymetrix.com/index.affx).

\section{Acknowledgments}

We thank Regina S. Whitaker, Lauren R. Simel, Tiffany Perry, Darby Kroyer, Carole Grenier, Cara E. Davis, and Kerone Walker for their technical contributions. This research was supported by grant W81XWH-05-1-0053 to S.K.M. from the Department of Defense CDMRP Ovarian Cancer Research Program and by grant 
21791551 to N.M. from the Japan Society for the Promotion of Science.

\section{References}

Ahuja N, Li Q, Mohan AL, Baylin SB, Issa JP. 1998. Aging and DNA methylation in colorectal mucosa and cancer. Cancer Res 58: 54895494.

Alvi AJ, Rader JS, Broggini M, Latif F, Maher ER. 2001. Microsatellite instability and mutational analysis of transforming growth factor beta receptor type II gene (TGFBR2) in sporadic ovarian cancer. Mol Pathol 54: 240-243.

Andrechek ER, Mori S, Rempel RE, Chang JT, Nevins JR. 2008. Patterns of cell signaling pathway activation that characterize mammary development. Development 135: 2403-2413.

Antequera F. 2003. Structure, function and evolution of CpG island promoters. Cell Mol Life Sci 60: 1647-1658.

Baba T, Convery PA, Matsumura N, Whitaker RS, Kondoh E, Perry T, Huang Z, Bentley RC, Mori S, Fujii S, et al. 2009. Epigenetic regulation of CD133 and tumorigenicity of CD133+ ovarian cancer cells. Oncogene 28: 209218.

Badgwell D, Bast RC Jr. 2007. Early detection of ovarian cancer. Dis Markers 23: $397-410$

Barton CA, Hacker NF, Clark SJ, O'Brien PM. 2008. DNA methylation changes in ovarian cancer: implications for early diagnosis, prognosis and treatment. Gynecol Oncol 109: 129-139.

Berchuck A, Iversen ES, Lancaster JM, Pittman J, Luo J, Lee P, Murphy S, Dressman HK, Febbo PG, West M, et al. 2005. Patterns of gene expression that characterize long-term survival in advanced stage serous ovarian cancers. Clin Cancer Res 11: 3686-3696.

Bild AH, Yao G, Chang JT, Wang Q, Potti A, Chasse D, Joshi MB, Harpole D, Lancaster JM, Berchuck A, et al. 2006. Oncogenic pathway signatures in human cancers as a guide to targeted therapies. Nature 439: 353-357.

Cairns P. 2009. 5'-Azacytidine expression arrays. Methods Mol Biol 507: 165174.

Chang JT, Nevins JR. 2006. GATHER: A systems approach to interpreting genomic signatures. Bioinformatics 22: 2926-2933.

Chen SJ, Ning H, Ishida W, Sodin-Semrl S, Takagawa S, Mori Y, Varga J. 2006 The early-immediate gene EGR-1 is induced by transforming growth factor-beta and mediates stimulation of collagen gene expression. J Biol Chem 281: 21183-21197.

Chen HY, Yu SL, Chen CH, Chang GC, Chen CY, Yuan A, Cheng CL, Wang $\mathrm{CH}$, Terng HJ, Kao SF, et al. 2007. A five-gene signature and clinical outcome in non-small-cell lung cancer. N Engl J Med 356: 11-20.

Ding W, Tang Q, Espina V, Liotta LA, Mauger DT, Mulder KM. 2005. A transforming growth factor-beta receptor-interacting protein frequently mutated in human ovarian cancer. Cancer Res 65: 6526-6533.

Do TV, Kubba LA, Du H, Sturgis CD, Woodruff TK. 2008. Transforming growth factor- $\beta 1$, transforming growth factor- $\beta 2$, and transforming growth factor- $\beta 3$ enhance ovarian cancer metastatic potential by inducing a Smad3-dependent epithelial-to-mesenchymal transition. Mol Cancer Res 6: 695-705.

Dunfield LD, Dwyer EJ, Nachtigal MW. 2002. TGF beta-induced Smad signaling remains intact in primary human ovarian cancer cells. Endocrinology 143: 1174-1181.

Esteller M. 2007. Cancer epigenomics: DNA methylomes and histonemodification maps. Nat Rev Genet 8: 286-298.

Francis-Thickpenny KM, Richardson DM, van Ee CC, Love DR, Winship IM, Baguley BC, Chenevix-Trench G, Shelling AN. 2001. Analysis of the TGF beta functional pathway in epithelial ovarian carcinoma. Br J Cancer $\mathbf{8 5}$ : 687-691.

Furuta J, Nobeyama Y, Umebayashi Y, Otsuka F, Kikuchi K, Ushijima T. 2006 Silencing of Peroxiredoxin 2 and aberrant methylation of $33 \mathrm{CpG}$ islands in putative promoter regions in human malignant melanomas. Cancer Res 66: 6080-6086.

Goh L, Murphy SK, Muhkerjee S, Furey TS. 2007. Genomic sweeping for hypermethylated genes. Bioinformatics 23: 281-288.

Havrilesky LJ, Hurteau JA, Whitaker RS, Elbendary A, Wu S, Rodriguez GC, Bast RC Jr, Berchuck A. 1995. Regulation of apoptosis in normal and malignant ovarian epithelial cells by transforming growth factor beta Cancer Res 55: 944-948.

Herfs M, Hubert P, Kholod N, Caberg JH, Gilles C, Berx G, Savagner P, Boniver J, Delvenne P. 2008. Transforming growth factor- $\beta 1$-mediated Slug and Snail transcription factor up-regulation reduces the density of Langerhans cells in epithelial metaplasia by affecting E-cadherin expression. Am J Pathol 172: 1391-1402.

Hirashima Y, Kobayashi H, Suzuki M, Tanaka Y, Kanayama N, Terao T. 2003. Transforming growth factor-beta1 produced by ovarian cancer cell line HRA stimulates attachment and invasion through an up-regulation of plasminogen activator inhibitor type- 1 in human peritoneal mesothelial cells. J Biol Chem 278: 26793-26802.

Hoque MO, Kim MS, Ostrow KL, Liu J, Wisman GB, Park HL, Poeta ML, Jeronimo C, Henrique R, Lendvai A, et al. 2008. Genome-wide promoter analysis uncovers portions of the cancer methylome. Cancer Res 68: 2661-2670.

Huang Z, Wen Y, Shandilya R, Marks JR, Berchuck A, Murphy SK. 2006. High throughput detection of M6P/IGF2R intronic hypermethylation and LOH in ovarian cancer. Nucleic Acids Res 34: 555-563.

Ibanez de Caceres I, Dulaimi E, Hoffman AM, Al-Saleem T, Uzzo RG, Cairns P. 2006. Identification of novel target genes by an epigenetic reactivation screen of renal cancer. Cancer Res 66: 5021-5028.

Ihn H. 2002. Pathogenesis of fibrosis: Role of TGF-beta and CTGF. Curr Opin Rheumatol 14: 681-685.

Kang SH, Bang YJ, Im YH, Yang HK, Lee DA, Lee HY, Lee HS, Kim NK, Kim SJ. 1999. Transcriptional repression of the transforming growth factor-beta type I receptor gene by DNA methylation results in the development of TGF-beta resistance in human gastric cancer. Oncogene 18: 7280-7286.

Karpf AR, Peterson PW, Rawlins JT, Dalley BK, Yang Q, Albertsen H, Jones DA. 1999. Inhibition of DNA methyltransferase stimulates the expression of signal transducer and activator of transcription 1,2, and 3 genes in colon tumor cells. Proc Natl Acad Sci 96: 14007-14012.

Keen JC, Garrett-Mayer E, Pettit C, Mack KM, Manning J, Herman JG, Davidson NE. 2004. Epigenetic regulation of protein phosphatase 2A (PP2A), lymphotactin (XCL1) and estrogen receptor alpha (ER) expression in human breast cancer cells. Cancer Biol Ther 3: 1304-1312.

Kondoh E, Mori S, Yamaguchi K, Baba T, Matsumura N, Cory Barnett J, Whitaker RS, Konishi I, Fujii S, Berchuck A, et al. 2010. Targeting slowproliferating ovarian cancer cells. Int J Cancer 126: 2448-2456.

Kwabi-Addo B, Chung W, Shen L, Ittmann M, Wheeler T, Jelinek J, Issa JP. 2007. Age-related DNA methylation changes in normal human prostate tissues. Clin Cancer Res 13: 3796-3802.

Li Q, Ahuja N, Burger PC, Issa JP. 1999. Methylation and silencing of the Thrombospondin-1 promoter in human cancer. Oncogene 18: 32843289.

Liang G, Gonzales FA, Jones PA, Orntoft TF, Thykjaer T. 2002. Analysis of gene induction in human fibroblasts and bladder cancer cells exposed to the methylation inhibitor 5-aza-2'-deoxycytidine. Cancer Res 62: 961-966.

Lounis H, Provencher D, Godbout C, Fink D, Milot MJ, Mes-Masson AM. 1994. Primary cultures of normal and tumoral human ovarian epithelium: A powerful tool for basic molecular studies. Exp Cell Res 215: 303-309.

Lynch MA, Nakashima R, Song H, DeGroff VL, Wang D, Enomoto T, Weghorst CM. 1998. Mutational analysis of the transforming growth factor beta receptor type II gene in human ovarian carcinoma. Cancer Res 58: 4227-4232.

Ma C, Rong Y, Radiloff DR, Datto MB, Centeno B, Bao S, Cheng AW, Lin F, Jiang S, Yeatman TJ, et al. 2008. Extracellular matrix protein betaigh3/TGFBI promotes metastasis of colon cancer by enhancing cell extravasation. Genes Dev 22: 308-321.

Massague J. 2008. TGF $\beta$ in cancer. Cell 134: 215-230.

Medici D, Hay ED, Olsen BR. 2008. Snail and Slug promote epithelialmesenchymal transition through beta-catenin-T-cell factor-4dependent expression of transforming growth factor-beta3. Mol Biol Cell 19: $4875-4887$.

Mund C, Brueckner B, Lyko F. 2006. Reactivation of epigenetically silenced genes by DNA methyltransferase inhibitors: Basic concepts and clinical applications. Epigenetics 1: 7-13.

Muthusamy V, Duraisamy S, Bradbury CM, Hobbs C, Curley DP, Nelson B, Bosenberg M. 2006. Epigenetic silencing of novel tumor suppressors in malignant melanoma. Cancer Res 66: 11187-11193.

Nilsson EE, Skinner MK. 2002. Role of transforming growth factor beta in ovarian surface epithelium biology and ovarian cancer. Reprod Biomed Online 5: 254-258.

Onwuegbusi BA, Aitchison A, Chin SF, Kranjac T, Mills I, Huang Y, LaoSirieix P, Caldas C, Fitzgerald RC. 2006. Impaired transforming growth factor beta signalling in Barrett's carcinogenesis due to frequent SMAD4 inactivation. Gut 55: 764-774.

Renzoni EA, Abraham DJ, Howat S, Shi-Wen X, Sestini P, Bou-Gharios G, Wells AU, Veeraraghavan S, Nicholson AG, Denton CP, et al. 2004. Gene expression profiling reveals novel TGF $\beta$ targets in adult lung fibroblasts. Respir Res 5: 24. doi: 10.1186/1465-9921-5-24.

Rodriguez GC, Haisley C, Hurteau J, Moser TL, Whitaker R, Bast RC Jr, Stack MS. 2001. Regulation of invasion of epithelial ovarian cancer by transforming growth factor-beta. Gynecol Oncol 80: 245-253.

Shames DS, Girard L, Gao B, Sato M, Lewis CM, Shivapurkar N, Jiang A, Perou CM, Kim YH, Pollack JR, et al. 2006. A genome-wide screen for promoter methylation in lung cancer identifies novel methylation markers for multiple malignancies. PLoS Med 3: e486. doi: 10.1371/ journal.pmed.0030486. 


\section{Matsumura et al.}

Suzuki MM, Bird A. 2008. DNA methylation landscapes: Provocative insights from epigenomics. Nat Rev Genet 9: 465-476.

Suzuki H, Gabrielson E, Chen W, Anbazhagan R, van Engeland M, Weijenberg MP, Herman JG, Baylin SB. 2002. A genomic screen for genes upregulated by demethylation and histone deacetylase inhibition in human colorectal cancer. Nat Genet 31: 141-149.

Tothill RW, Tinker AV, George J, Brown R, Fox SB, Lade S, Johnson DS, Trivett MK, Etemadmoghadam D, Locandro B, et al. 2008. Novel molecular subtypes of serous and endometrioid ovarian cancer linked to clinical outcome. Clin Cancer Res 14: 5198-5208.

Ueki T, Walter KM, Skinner H, Jaffee E, Hruban RH, Goggins M. 2002. Aberrant CpG island methylation in cancer cell lines arises in the primary cancers from which they were derived. Oncogene 21: 2114-2117.

Umetani N, Mori T, Koyanagi K, Shinozaki M, Kim J, Giuliano AE, Hoon DS. 2005. Aberrant hypermethylation of ID4 gene promoter region increases risk of lymph node metastasis in T1 breast cancer. Oncogene 24: 4721-4727.

Ushijima T. 2005. Detection and interpretation of altered methylation patterns in cancer cells. Nat Rev Cancer 5: 223-231.

Wen XZ, Akiyama Y, Baylin SB, Yuasa Y. 2006. Frequent epigenetic silencing of the bone morphogenetic protein 2 gene through methylation in gastric carcinomas. Oncogene 25: 2666-2673.
West M, Blanchette C, Dressman H, Huang E, Ishida S, Spang R, Zuzan H, Olson JA Jr, Marks JR, Nevins JR. 2001. Predicting the clinical status of human breast cancer by using gene expression profiles. Proc Natl Acad Sci 98: $11462-11467$

Yamashita S, Tsujino Y, Moriguchi K, Tatematsu M, Ushijima T. 2006. Chemical genomic screening for methylation-silenced genes in gastric cancer cell lines using 5-aza-2'-deoxycytidine treatment and oligonucleotide microarray. Cancer Sci 97: 64-71.

Yoo CB, Jones PA. 2006. Epigenetic therapy of cancer: Past, present and future. Nat Rev Drug Discov 5: 37-50.

Yu H, Konigshoff M, Jayachandran A, Handley D, Seeger W, Kaminski N, Eickelberg O. 2008. Transgelin is a direct target of TGF-beta/Smad3dependent epithelial cell migration in lung fibrosis. FASEB J 22: 1778 1789.

Zhao H, Shiina H, Greene KL, Li LC, Tanaka Y, Kishi H, Igawa M, Kane CJ, Carroll P, Dahiya R. 2005. CpG methylation at promoter site -140 inactivates TGFbeta 2 receptor gene in prostate cancer. Cancer 104: 44-52.

Received April 6, 2010; accepted in revised form November 9, 2010.

\section{Genome Research}




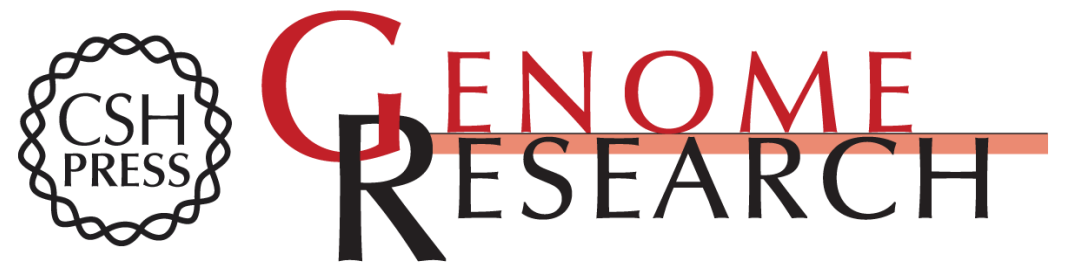

\section{Epigenetic suppression of the TGF-beta pathway revealed by transcriptome profiling in ovarian cancer}

Noriomi Matsumura, Zhiqing Huang, Seiichi Mori, et al.

Genome Res. 2011 21: 74-82 originally published online December 14, 2010

Access the most recent version at doi:10.1101/gr.108803.110

Supplemental Material

References

License

Email Alerting Service
http://genome.cshlp.org/content/suppl/2010/11/11/gr.108803.110.DC1

This article cites 58 articles, 22 of which can be accessed free at: http://genome.cshlp.org/content/21/1/74.full.html\#ref-list-1

Receive free email alerts when new articles cite this article - sign up in the box at the top right corner of the article or click here.

\section{Affordable, Accurate Sequencing.}

To subscribe to Genome Research go to:

https://genome.cshlp.org/subscriptions 